\title{
La formación para la investigación como eje curricular en la formación docente: el caso de la Carrera de Educación Preescolar de la UNED-Costa Rica
}

Recibido: 20 de junio de 2011

\section{RESUMEN}

Este artículo presenta resultados de una evaluación curricular realizada en una carrera de Educación Preescolar; la valoración se centra en la formación para la investigación. Se determina que $62.2 \%$ de los cursos del plan de estudios analizado incluye actividades investigativas dirigidas a visitas, diseño y ejecución de actividades educativas en centros escolares. Pese a que esas actividades en la mayoría de las asignaturas son precedidas por una investigación diagnóstica (estudiantes, grupo o centros educativos) no se visualiza ese componente investigativo en las instrucciones. Así, la minoría de las asignaturas explicita que debe realizar un trabajo de investigación como parte de sus actividades académicas. En la carrera se evidencia la necesidad de determinar el tipo de actividades investigativas a realizar por los estudiantes, así como la importancia de fortalecer algunas actividades como la investigación bibliográfica, la rigurosidad en el uso de fuentes bibliográficas y la prevención del plagio en todas las asignaturas para apoyar la formación profesional del estudiante.

\section{PALABRAS CLAVE}

Formación para investigación, Educación Preescolar, Formación docente en Preescolar
Aprobado: 4 de octubre de 2011

\section{SUMMARY}

This paper presents the results of a curricular assessment, related to the Preschool Education degree and focused on research training. It shows that $62.2 \%$ of the courses within the analyzed curriculum include research activities as visits, design and implementation of educative activities in educational centers. Although those activities in most of the subjects are preceded by a diagnostic research (students, groups, and educational centers) that research component cannot be found within the instructions. Thus, only a few of the subjects in the curriculum require some research as a part of their academicals activities. Therefore it is important for the Preschool degree to define which types of research activities should be done by the students, according to the desired professional profile. There are also some activities including bibliographic research, the rigor on using bibliographical sources, and information to avoid plagiarism, which should be reinforced within all the subjects, in order to support the professional education of the student.

\section{KEY WORDS}

Research training, Preschool Education, Preschool teaching training 


\section{INTRODUCCIÓN}

El desarrollo de competencias investigativas en los profesionales en Educación es una acción vital en los procesos de formación inicial (Campos y Castro, 2010; Campos y Chinchilla, 2009; Venegas, 2009; Alfaro y Francis, 2009). Saber investigar es esencial para el buen desempeño docente (Day, 2005; Freire, 2004; Stenhouse, 2003).

En la UNED, la investigación ha sido reconocida como una acción por fortalecer. Durante los últimos quinquenios, se ha generado una importante cantidad de políticas y normas orientadas a incentivar la investigación dentro de la institución y dentro de las unidades académicas (Morales, 2011). Asimismo, la investigación ha sido establecida como el $5^{\circ}$ eje curricular en el Plan Académico 2008 -2011, según acta 1656-2003 del Consejo Universitario, (UNED, 2008, p.31). Además, en el Modelo Pedagógico se establece la investigación como elemento base para la sostenibilidad y adecuación permanente de los procesos pedagógicos de la institución (UNED, 2004, p. 45). Por su parte, la Escuela de Ciencias de la Educación (ECE) de la UNED ha considerado la investigación como una de las acciones prioritarias del quehacer académico y su fortalecimiento ha sido impulsado a la luz de los siguientes procesos:

1. Los planes de estudio de todas las carreras de la ECE están pasando por un rediseño y la demanda de los cursos de investigación está cambiando, al igual que los requerimientos para los nuevos cursos que integren los planes de estudio remozados. Entre los perfiles de salida de las carreras de la ECE se contemplan rasgos o competencias que suponen formación para la investigación.

2. Los procesos de autoevaluación con miras a la acreditación que están desarrollando las carreras de la ECE exigen una articulación de la docencia, la investigación y la extensión en el quehacer académico de las cátedras y los programas.
3. La ECE requiere apoyo para la construcción de una cultura investigativa que permita la producción de conocimiento en materia educativa, dirigida a enriquecer las carreras y cumplir con la responsabilidad social de producir y gestionar el conocimiento relevante para contribuir a orientar el sistema educativo y la política pública en materia de Educación.

Se debe abordar el tema de la formación para la investigación porque en Costa Rica se ha detectado la necesidad de incrementar la producción investigativa en el sector educativo. Existe consenso en que se deben fortalecer las competencias investigativas de los profesionales en Educación, como una de las formas de inducir el cambio en un sistema educativo nacional que parece poco acorde con las necesidades y demandas sociales (Alfaro y Francis, 2009; Campos y Chinchilla, 2009; Venegas, 2008).

En el campo de la Educación Preescolar, la formación para la investigación es un tema de relevancia: la atención de la primera infancia significa la primera experiencia educativa del niño en el sistema formal y es justamente en la edad preescolar cuando se establecen los cimientos para el desarrollo y la formación de la personalidad, gracias a la plasticidad del cerebro, que posibilita conexiones que permiten el registro, asimilación y fijación de las estimulaciones del mundo que rodea al niño. Según la literatura en el campo, la mitad del potencial intelectual se desarrolla hasta los cuatro años y el estímulo ambiental que recibe el ser humano en este período crítico impacta el desarrollo mental, emocional y físico (Acevedo, 2007; AMEI, 2006, p. 12).

Los niños y las niñas que tienen la oportunidad de participar en un proceso educativo anterior a la escuela tienen mayores posibilidades de seguir estudios más largos, hay menos repitencia, deserción y sobre edad en los niveles siguientes y parecen tener mejores bases para insertarse más favorablemente a la sociedad 
(Comisión de las Comunidades Europeas, citado por Egido, 2000; Acevedo, 2007).

Así, mediar el proceso pedagógico en virtud del infante preescolar requiere facilitar su desarrollo cognitivo-lingüístico, socioemocional, psicomotriz, incluyendo sus derechos, la satisfacción de sus necesidades e intereses, el respeto por el contexto y sus componentes: axiológico, social, cultural, ambiental, histórico de pertenencia (Hidalgo y otras, 2010).

Aparte de la importancia de la educación en la edad preescolar para potenciar el desarrollo y el éxito escolar de las personas, subyace una condición de derechos que tienen las personas menores de edad de recibir educación. De allí la importancia de garantizar el acceso a la Educación Preescolar y de velar porque sea de calidad.

La actividad docente es uno de los factores que determina la calidad educativa; por ello, la importancia de lograr un perfil profesional pertinente para atender las necesidades educativas de las población de o a 6 años de edad.

Por tanto, la carrera de Educación Preescolar de la UNED ha sido rediseñada. Actualmente, su plan de estudios se asienta en cuatro áreas formativas: formación humanística, mediación pedagógica (dirigida a facilitar el aprendizaje en la niñez menor de 6 años mediante estrategias de aprendizaje lúdicas, placenteras, significativas, novedosas y atractivas), formación en desarrollo humano (conocimiento de las diferentes etapas de crecimiento y desarrollo del infante) y un área complementaria mediante la cual desarrolla conocimientos, destrezas y habilidades en campos como la investigación y tecnologías informáticas, que le "permiten dar respuesta a las necesidades y demandas que surjan en su campo de acción, promoviendo un profesional con formación sólida y con una visión más amplia sobre su labor docente" (Hidalgo y otras, 2010 p. 106).

En consonancia con las áreas de formación expuestas, el docente de preescolar debe cumplir con algunas funciones que lo hacen un profesional "polivalente, abierto y dinámico" (Ministerio de Educación y Deportes, 2005a), como se observa en la Figura 1.

El profesional en preescolar debe ser un docente capaz de reflexionar en torno a su actuación y de poseer criterios que le ayudan a mejorar su labor. Además, si es un investigador, va a tener apertura para incorporar "en el ámbito de la Educación Inicial, habilidades, conocimientos y actitudes para diseñar, desarrollar, evaluar y formular estrategias y programas de intervención educativa en contextos socio-educativos y culturales cambiantes" (Ministerio de Educación y Deportes, 2005a, p. 67), lo que lo convierte en un planificador de su labor pedagógi$c a$, a partir de la realidad del infante.

En este sentido, tal como lo plantea Siverio $(2008$, p. 26) no se pretende que el docente de preescolar sea un cientíico, pero sí que logre conocer a profundidad a sus estudiantes, y que a partir de una investigación diagnóstica logre planear y ejecutar propuestas educativas pertinentes y potenciadoras de aprendizajes significativos.

Asimismo, debe ser un profesional autoreflexivo capaz de repensar críticamente su quehacer docente (Pruzzo, 2002) y actuar en favor del mejoramiento de sus prácticas pedagógicas y la calidad educativa (Siverio, 2008; Sevillano, Pascual y Crespo, 2007; Mcmillan y Schummacher, 2005). Esta relación entre las actividades investigativas y la función pedagógica de los docentes ha sido sintetizada detalladamente por Cerda (2007), tal como se observa en el Cuadro 1.

Al formar docentes investigadores para la atención de preescolares, es necesario el análisis de la estructura curricular de la carrera de Educación Preescolar de la UNED. Se deben identificar las características de la formación para la investigación que se está desarrollando en las asignaturas que conforman el plan de estudio y valorar las estrategias de formación para la investigación utilizadas a lo largo del proceso 


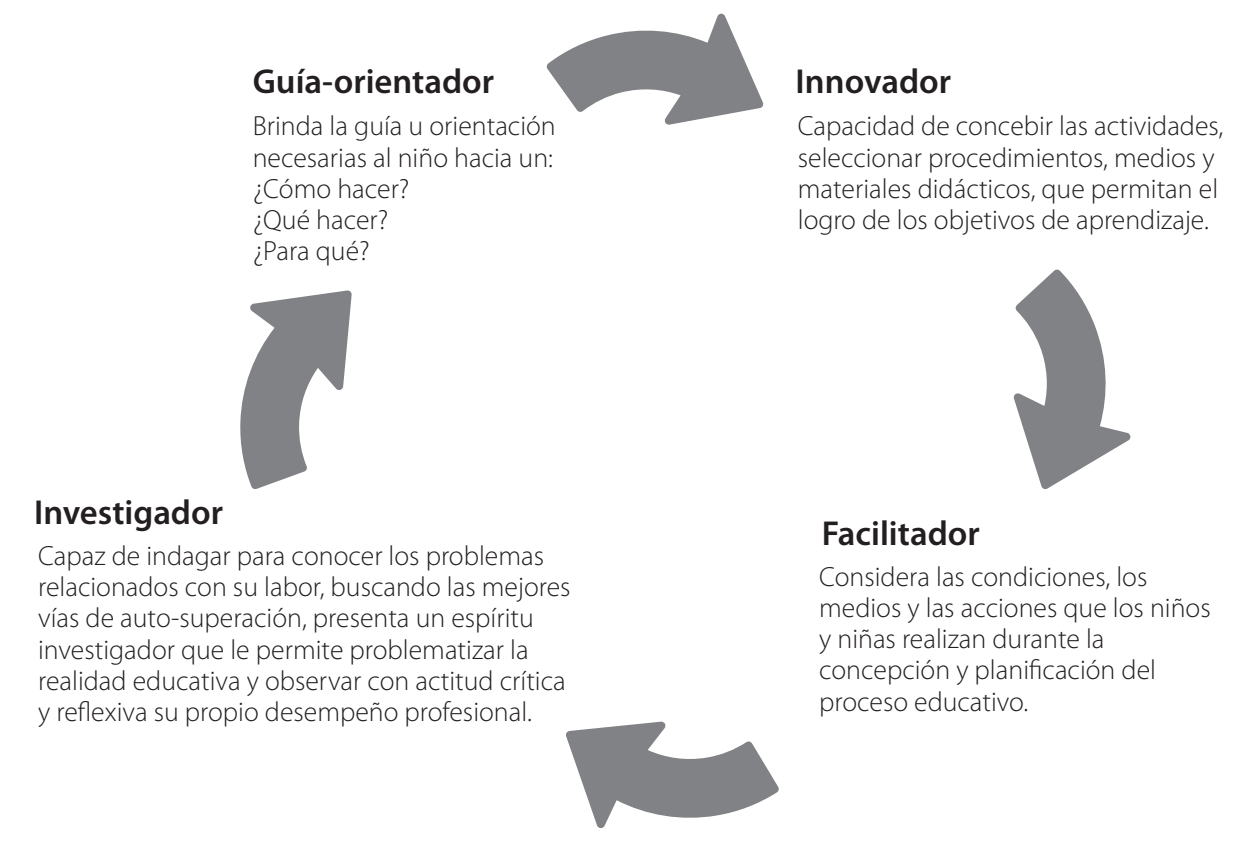

Figura 1. Competencias clave del docente de educación preescolar.

Fuente: Adaptado de Siverio (2008)

formativo. A partir de ese análisis, se puede establecer nuevas acciones que favorezcan el desarrollo de la competencia investigativa en los futuros docentes $y$, con ello, contribuir al mejoramiento de su labor pedagógica y de la atención a las personas menores de seis años y sus familias.

\section{METODOLOGÍA}

Se analizó el plan de estudios de la carrera de Bachillerato y Licenciatura en Educación Preescolar de la Universidad Estatal a Distancia de Costa Rica (UNED), para determinar en qué medida la investigación constituye un eje curricular y cuáles serían las estrategias para mejorar esa formación. Se analizaron todas las Orientaciones de curso de 48 asignaturas; 44 del plan de estudios vigente durante el último cuatrimestre del 2010 y cuatro correspondientes a asignaturas optativas.
Para extraer información que permitiera identificar la formación para la investigación, en cada una de las asignaturas se utilizó las siguientes categorías: ejercicios investigativos en la evaluación sumativa de proyectos de investigación u otra actividad que involucrará la aplicación de una técnica de recolección o sistematización de información. También se observó si en las orientaciones del curso se le brinda inducción al estudiante acerca de:

"Las bases de datos con las revistas electrónicas a las que tiene acceso la UNED.

»El uso de alguna guía de estilo o sistema de citación para indicar al estudiante cómo debe realizar las referencias dentro del texto y en la notación de la bibliografía consultada en sus trabajos académicos.

»El concepto de plagio, su prevención y la normativa institucional referente a este tema. 
Cuadro 1. Capacidades y atributos indispensables para la actividad pedagógica e investigativa.

\begin{tabular}{|l|l|}
\hline \multicolumn{2}{|c|}{ Capacidades ó Atributos } \\
\hline Capacidad de aprender. & $\begin{array}{l}\text { Lógica y razonamiento inductivo-deductivo y de } \\
\text { simulación. }\end{array}$ \\
\hline $\begin{array}{l}\text { Capacidad de aplicar los conocimientos en la } \\
\text { práctica. }\end{array}$ & $\begin{array}{l}\text { Pensamiento crítico y la capacidad de definir y } \\
\text { resolver problemas. }\end{array}$ \\
\hline Capacidad de análisis y síntesis. & Creatividad y curiosidad. \\
\hline Capacidad de adaptarse a las nuevas situaciones. & Capacidad para hacer preguntas. \\
\hline Habilidades interpersonales. & Capacidad de la observación y la indagación. \\
\hline Creatividad. & $\begin{array}{l}\text { Identificación de problemas relacionados con el } \\
\text { objeto de estudio. }\end{array}$ \\
\hline Comunicación oral y escrita. & Búsqueda de información. \\
\hline Toma de decisiones. & $\begin{array}{l}\text { Tratamiento, interpretación y evaluación de la } \\
\text { información. }\end{array}$ \\
\hline Capacidad crítica y autocrítica. & Prácticas multi, inter y transdiciplinarias. \\
\hline Capacidad básica del manejo de la computadora. & Capacidad de autodefinición del trabajo. \\
\hline Capacidad de trabajo en equipo interdisciplinario. & Anticipación al análisis de riesgo. \\
\hline $\begin{array}{l}\text { Conocimientos generales y básicos del tema en } \\
\text { estudio. }\end{array}$ & Prospectiva. \\
\hline Compromiso ético. & Conocimiento de una segunda lengua. \\
\hline Conocimiento básico de la profesión. & Apreciación de la diversidad y multiculturalidad. \\
\hline Habilidad de investigación. & \\
\hline
\end{tabular}

Fuente: Cerda (2007).

$\gg$
La divulgación de líneas de investigación y articulación de la investigación desa- rrollada por académicos y estudiantes.

Para cada una de esas categorías se identificaron algunos indicadores que permitieron cuantificar la presencia de la investigación en las asignaturas. Asimismo, se lograron identificar las estrategias o actividades de aprendizaje utilizadas para formar a los estudiantes tanto en investigación o en otras competencias, pero que tienen como valor agregado la formación de competencias investigativas. A partir de la información, se analizan los datos y se relaciona con literatura existente en el campo de la formación de docentes para la investigación. Luego se elabora un conjunto de recomendaciones que permitirá mejorar la formación para la investigación de sus estudiantes.

\section{RESULTADOS}

En la ECE, la formación para la investigación se brinda mediante las asignaturas que imparte la Cátedra de Investigación Educativa. Se centra en la enseñanza de la metodología de la investigación sin que previa o paralelamente haya competencias básicas para establecer el juicio crítico y analítico y la capacidad de problematización, elementos clave para formar en investigación al futuro docente. De igual manera, no se cuenta con un plan de transversalización de la investigación a fin de formar al estudiante de forma organizada, progresiva y sistemática, para ser un docente de Educación Preescolar capaz de investigar acerca y en su quehacer.

Los cursos de investigación en el plan de estudios le permiten al estudiante profundizar en 
el estudio de los contenidos sobre teoría y metodología de la investigación. Tendrán conocimientos, habilidades y destrezas para desarrollar ejercicios académicos de investigación.

Sin embargo, esas competencias no se logran únicamente en los cursos de investigación. Por el contrario, tal como lo señala Restrepo (2004) aprender a investigar requiere la exposición reiterada del estudiante a procesos y ejercicios investigativos y a dinámicas de observación y análisis crítico de las realidades en las que se encuentra y de su propio quehacer, en todas las asignaturas del plan de estudios (Figura 2).

Asumir la investigación como eje curricular en la formación es cambiar de paradigma: nos obliga a repensar cómo se está formando en esas asignaturas, las cuales están engranadas y articuladas con el resto del plan. No es que en todas las asignaturas se estudien contenidos de investigación, sino que en un número importante de materias se contribuya a formar a los estudiantes utilizando la investigación como un medio pedagógico, recurso didáctico o estrategia de aprendizaje.

A la luz de la revisión de las orientaciones de los cursos incluidos en el plan de estudios, se observa que $62 \%$ de las asignaturas incluidas en el plan de estudios tiene ejercicios investigativos entre las actividades académicas. En términos de asignación de carga académica, las actividades que suponen el desarrollo de algunas acciones investigativas se presentan en cerca de 39 de los 174 créditos que integran el plan de estudios, tal como se observa en el Cuadro 2.

En las asignaturas con actividades de acción investigativa (como porcentaje de la evaluación

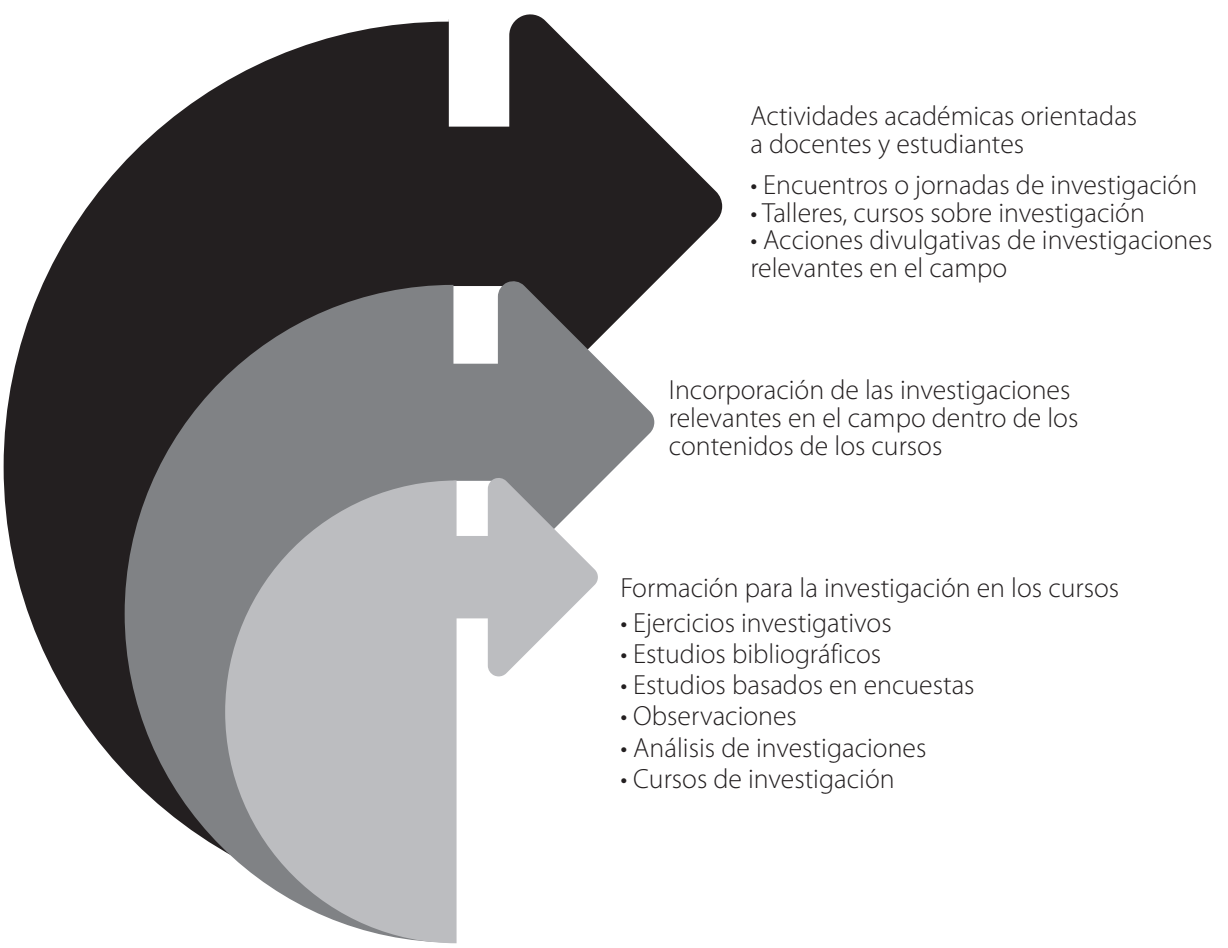

Figura 2. Incorporación de la investigación en las diferentes dimensiones del curriculum de la carrera.

Fuente: Elaboración propia de las investigadoras. 
sumativa), el valor respecto de la calificación total del curso que se le asigna es (para la mayoría de los casos) 30\% o 40\%. Solo en dos asignaturas se le atribuye más de un $50 \%$ (ver Cuadro 3).

Las acciones o trabajos investigativos en la evaluación sumativa de las materias están principalmente orientadas a la recolección de información mediante observación, entrevistas y revisiones bibliográficas. La mayoría de esas acciones se realiza en el Diplomado (ver Cuadro 4).
Los estudiantes visitan los centros educativos durante el Diplomado; así aprecian los potenciales espacios profesionales, consolidan su decisión vocacional e integran teoría y práctica. No obstante, en Bachillerato y Licenciatura esas actividades investigativas disminuyen considerablemente cuando se esperaría que los estudiantes se enfrenten a ejercicios y problemas de investigación más complejos mediante diversos enfoques y herramientas metodológicas para desarrollar posteriormente el trabajo final de graduación (TFG) y

Cuadro 2. Distribución de asignaturas que contienen actividades que suponen acciones investigativas.

\begin{tabular}{|l|c|c|c|c|c|}
\hline \multicolumn{1}{|c|}{ Nivel } & $\begin{array}{c}\text { Cantidad } \\
\text { total de } \\
\text { créditos }\end{array}$ & $\begin{array}{c}\text { Cantidad de } \\
\text { total de } \\
\text { asignaturas }\end{array}$ & $\begin{array}{c}\text { Asignaturas que } \\
\text { incluyen acciones } \\
\text { investigativas }\end{array}$ & $\begin{array}{c}\text { Cantidad de créditos } \\
\text { destinados a las acti- } \\
\text { vidades que incluyen } \\
\text { acciones investigativas }\end{array}$ & $\begin{array}{c}\text { Porcentaje de } \\
\text { los créditos** }\end{array}$ \\
\hline Diplomado & 73 & 16 & 10 & 13.3 & 18.2 \\
\hline Bachillerato & 67 & 18 & 12 & $15 \cdot 3$ & 22.8 \\
\hline Licenciatura & 34 & 11 & 6 & 9.3 & 27.3 \\
\hline TOTAL & $174^{*}$ & 45 & 28 & 37.9 & 68.3 \\
\hline
\end{tabular}

*Para el nivel de Diplomado son 70 créditos, pero se incluye la asignatura optativa. Y para el Bachillerato son 68; sin embargo, se quita la práctica y se agrega la electiva. En Licenciatura, son 31, pero tomando en cuenta las electivas son 34 créditos.

** Se tomó como parámetro el porcentaje de la evaluación sumativa que representan las actividades de aprendizaje que suponen alguna acción de investigación en cada asignatura.

Fuente: Elaboración propia de las investigadoras.

Cuadro 3. Porcentaje de la evaluación sumativa atribuida a asignaciones o trabajos que involucran alguna acción investigativa.

\begin{tabular}{|c|c|c|c|}
\hline \multirow{2}{*}{ Porcentaje asignado } & & Cantidad de asignaturas & \\
\cline { 2 - 4 } & Diplomado & Bachillerato & Licenciatura \\
\hline $20 \%$ & - & - & 1 \\
\hline $25 \%$ & - & 5 & 3 \\
\hline $30 \%$ & 5 & - & - \\
\hline $40 \%$ & 4 & 3 & 1 \\
\hline $50 \%$ & 1 & 1 & - \\
\hline $60 \%$ & - & - & 1 \\
\hline
\end{tabular}

Fuente: Elaboración propia de las investigadoras. 
Cuadro 4. Acciones investigativas incluidas en los trabajos o asignaciones según subproceso investigativo.

\begin{tabular}{|c|c|c|c|}
\hline Actividades o técnicas empleadas & Diplomado & Bachillerato & Licenciatura \\
\hline \multicolumn{4}{|l|}{ Diseño de instrumentos } \\
\hline Guía de observación & -- & 1 & -- \\
\hline Guía de entrevista & -- & 1 & -- \\
\hline \multicolumn{4}{|l|}{ Recolección de datos } \\
\hline Entrevista estructurada & 2 & 1 & -- \\
\hline Entrevista no estructurada & 3 & 4 & 1 \\
\hline Observación & 6 & 6 & 3 \\
\hline Encuesta & -- & -- & 1 \\
\hline Revisión bibliográfica & 4 & 3 & 3 \\
\hline Revisión documental & 2 & 1 & -- \\
\hline \multicolumn{4}{|l|}{ Análisis de la información } \\
\hline Análisis de contenido & 2 & 3 & -- \\
\hline Estudio de caso & -- & 1 & 1 \\
\hline Análisis de datos obtenidos & 2 & 3 & -- \\
\hline \multicolumn{4}{|c|}{ Sistematización y registro de la información } \\
\hline Elaboración de informes & 3 & 4 & 1 \\
\hline Elaboración de reportes & 1 & 3 & 1 \\
\hline Registro anecdótico & 1 & -- & -- \\
\hline \multicolumn{4}{|l|}{ Socialización de la información } \\
\hline Charla & -- & 1 & -- \\
\hline Taller & --- & 1 & -- \\
\hline
\end{tabular}

Fuente: Elaboración propia de las investigadoras.

ser profesionales capaces de investigar y elaborar propuestas pedagógicas a partir de los resultados de la investigación.

En la carrera, las acciones investigativas están concentradas fundamentalmente en diagnósticos. No se recurre a otros tipos de investigación como la evaluativa o la prospectiva. En pocas asignaturas se induce a utilizar los resultados de un ejercicio investigativo para proponer una intervención y modificar la realidad investigada. Solo en Bachillerato (4) y Licenciatura (3) se orienta al estudiante a realizar tal enlace entre investigación e intervención, pues debería asimilar la relación investigación-acción como un continuo natural y mejorar así su práctica pedagógica.

En la misma línea, como lo plantean Campos y Castro (2010), a fin de mejorar la formación para la investigación en los futuros docentes, hay que ampliar el proceso de investigación que tradicionalmente se les ha enseñado, incluir los resultados de las investigaciones y mejorar las propuestas pedagógicas en educación, aplicar los resultados en la transformación del fenómeno investigado y evaluar los efectos de la incorporación de tales resultados.

Entonces, habría que dar "énfasis a diseños de investigación orientados al cambio, que 
permita trascender la investigación diagnóstica, exploratoria-descriptiva hacia la investigación aplicada o investigación transformadora e ir construyendo condiciones académicas suficientes para desarrollar investigación pronóstica en la que se vislumbren nuevos escenarios proyectados a futuro. En este sentido, la investigación acción en los cursos prácticos y la práctica profesional puede constituirse en una excelente estrategia formativa" (Campos y Castro, 2010, p. 116).

En adición al tema anterior, se encontró que en la mayoría de las asignaturas se ofrece a los estudiantes información de las bases de datos de revistas electrónicas a las cuales la UNED está suscrita. Sin embargo, en casi ninguno de los cursos se les induce ni se les explica cómo utilizarlas para apoyar alguna investigación bibliográfica o fundamentar una propuesta pedagógica.

Con respecto a la forma correcta de realizar referencias y citas dentro del texto, solamente en nueve asignaturas se le indica al estudiante el formato que debe utilizar. De esas asignaturas, solo dos están en el nivel de Diplomado, cuando más instrucción necesita el estudiante respecto de este requerimiento académico. Solamente en el $9 \%$ de las asignaturas se le informa al estudiante con respecto al plagio y la normativa institucional relacionada con el mismo, lo cual parece ser insuficiente para informar debidamente a la población estudiantil y minimizar el riesgo de incidencia.

Adicionalmente en doce de los cursos del plan se brinda información de la programación de "Onda UNED". Resultaría muy conveniente, con miras a ampliar estos y otros recursos en línea de la UNED, si en las actividades de la evaluación sumativa, se induce al estudiante a escuchar un programa específico, transmitido por "Onda UNED", mediante el acceso desde Internet.

De las asignaturas del plan de estudios, diez se desarrollan con algún grado de virtualidad: cuatro son híbridos y seis, bimodales. Esto es fundamental porque permite utilizar las tecnologías de la información y la comunicación (TIC) para que los estudiantes investiguen en red y usen recursos digitales disponibles en la red mundial para enriquecer los cursos.

A partir del análisis realizado, se evidencia la necesidad de organizar y diversificar el tipo de actividades y estrategias pedagógicas que se utilicen en las asignaturas del plan de estudios para fortalecer las competencias investigativas en los estudiantes de la carrera. En especial, aquellas competencias elementales que son compartidas por el quehacer investigativo y el quehacer docente, según Cerda (2007), y que se expresan en la Figura 1.

Al seguir los planteamientos de Cerda (2007) se encuentra el perfil de competencias investigativas establecido por Campos y Castro (2010), del cual se extraen algunas competencias investigativas básicas que permiten orientar la formación para la investigación en los distintos niveles de la carrera de Educación Preescolar, tal como se observa en el Cuadro 6.

Entre las estrategias y actividades de aprendizaje más utilizadas en las asignaturas analizadas,

Cuadro 5. Orientaciones básicas para la búsqueda y uso de fuentes bibliográficas.

\begin{tabular}{|l|c|c|c|}
\hline \multicolumn{1}{|c|}{ Actividades o técnicas empleadas } & Diplomado & Bachillerato & Licenciatura \\
\hline Uso bases de datos & 12 & 11 & 4 \\
\hline Uso de normas de estilo de citación & 2 & 4 & 3 \\
\hline Información para la prevención del plagio & 7 & 6 & 4 \\
\hline
\end{tabular}

Fuente: Elaboración propia de las investigadoras. 
Cuadro 6. Grado de intensidad en la formación de cada una de las competencias investigativas básicas según nivel en el plan de estudios.

\begin{tabular}{|c|c|c|c|c|c|c|c|c|c|}
\hline Competencias investigativas & & lom & & Bac & hille & & Lice & ncia & \\
\hline Capacidad para... & $\stackrel{\circ}{\frac{\rho}{m}}$ & $\frac{\circ}{\frac{0}{0}}$ & $\stackrel{\circ}{\frac{0}{4}}$ & $\stackrel{\circ}{\stackrel{0}{\oplus}}$ & $\frac{\circ}{\frac{0}{0}}$ & $\stackrel{\circ}{\frac{9}{4}}$ & $\stackrel{\circ}{\stackrel{\circ}{\circ}}$ & $\frac{\circ}{\frac{0}{g}}$ & $\frac{9}{4}$ \\
\hline Problematizar la realidad educativa. & & & $\mathrm{X}$ & & & $\mathrm{X}$ & & & $X$ \\
\hline $\begin{array}{l}\text { Identificar cuerpos teóricos relacionados con su práctica pedagó- } \\
\text { gica y el tema de investigación, extraer e integrar los elementos } \\
\text { que permitan enriquecer su labor como docente investigador. }\end{array}$ & $x$ & & & & $x$ & & & & $x$ \\
\hline $\begin{array}{l}\text { Plantear y ejecutar estrategias investigativas para responder a } \\
\text { los problemas de investigación y solventar los vacíos de conoci- } \\
\text { miento como parte praxis pedagógica. }\end{array}$ & $x$ & & & & $x$ & & & & $x$ \\
\hline $\begin{array}{l}\text { Hacer búsquedas efectivas de información en las diferentes } \\
\text { fuentes para responder al problema de investigación. }\end{array}$ & & & $x$ & & & $x$ & & & $x$ \\
\hline $\begin{array}{l}\text { Analizar la información y vincularla con el problema de investiga- } \\
\text { ción y las teorías existentes relacionadas con el problema. }\end{array}$ & $x$ & & & & $x$ & & & & $x$ \\
\hline $\begin{array}{l}\text { Analizar e interpretar la información obtenida para resolver el } \\
\text { problema de investigación. }\end{array}$ & $x$ & & & & $x$ & & & & $x$ \\
\hline $\begin{array}{l}\text { Sistematizar las experiencias pedagógicas de la práctica cotidia- } \\
\text { na reflexionar sobre ella, evaluarla y derivar problemas de inves- } \\
\text { tigación o acciones de mejoramiento. }\end{array}$ & & $x$ & & & & $x$ & & & $x$ \\
\hline $\begin{array}{l}\text { Hacer devolución de resultados de su investigación de forma oral } \\
\text { y escrita. }\end{array}$ & $X$ & & & & & $x$ & & & $x$ \\
\hline Gestionar conocimiento. & $x$ & & & & & $X$ & & & $x$ \\
\hline $\begin{array}{l}\text { Vincular los resultados de investigaciones a su práctica pedagó- } \\
\text { gica para innovarla. }\end{array}$ & & $x$ & & & & $x$ & & & $x$ \\
\hline
\end{tabular}

Fuente: Adaptado de Campos y Castro (2010).

que contribuyen a la formación para la investigación de los estudiantes, se encuentran:

1. Visitas a centros educativos para la observación (de un estudiante o de un grupo) y entrevistas con docentes o con padres o madres de familia.

2. Estudios de caso (de un estudiante o de un grupo) y diseño de un plan pedagógico para atender las necesidades del caso específico.

3. Investigaciones bibliográficas o revisiones de literatura en torno a diversos temas afines con la asignatura en la que se solicita.

4. Diseño y ejecución de proyectos de investigación de diversos temas asociados a la Educación Preescolar.

Pese a que las estrategias indicadas son pertinentes, conviene organizar este tipo de ejercicios y explorar la utilización de portafolios en los que los estudiantes tengan oportunidad de registrar reflexiones acerca de su proceso de aprendizaje; además, es útil el empleo de bitácoras de investigación, el uso del cuaderno 
de campo para la realización de observaciones participantes en visitas a los centros educativos. Conviene también enriquecer las asignaturas con la incorporación del material de estudio, de un conjunto de artículos científicos actualizados en el campo e informes de investigación. Para analizarlos críticamente, se debe complementar con guías de análisis; el objetivo es derivar información clave para el mejoramiento de la práctica pedagógica en Educación Preescolar.

Por otra parte, la revisión de literatura debe implementarse en la mayoría de las asignaturas como estrategia de aprendizaje clave para que el estudiante ejercite la búsqueda adecuada de información y fuentes de consulta y para que también mejore la redacción de informes.

Para el caso de los cursos que se desarrollan en línea, es valiosa la utilización de wikis, Google docs y otras herramientas de la web 2.0, para que los estudiantes desarrollen proyectos de investigación en grupos y así favorecer, adicionalmente, la capacidad de trabajo en equipo.

\section{CONCLUSIONES Y RECOMENDACIONES}

»En la carrera de Educación Preescolar existen muchos cursos que integran actividades de aprendizaje con alguna acción investigativa. Sin embargo, no se explicitan como tales en las orientaciones de los cursos y los objetivos de esas actividades no están dirigidos a la formación para la investigación; aparte, el componente investigativo no es el sustantivo en la mayoría de las actividades de aprendizaje, sino que el énfasis está en el planeamiento y la ejecución de actividades pedagógicas.

" La investigación debe constituirse en uno de los elementos clave en los procesos de formación de los futuros docentes de Educación Preescolar. Para ello, se requiere una resignificación del quehacer de la carrera, con la meta de ubicar la investigación como piedra angular de los procesos formativos y construir una cultura de investigación que comprenda todas las manifestaciones de la vida académica (Restrepo, 2004).

» En una cantidad relevante de asignaturas, las instrucciones brindadas a los estudiantes son imprecisas en cuanto a la denominación de métodos, técnicas e instrumentos de investigación. Eso podría confundir al estudiante; entonces, hay que unificar la denominación asignada a este tipo de elementos. Sería deseable que en la ECE se cuente con un glosario de términos de investigación educativa, accesible para los estudiantes.

"En todos los cursos analizados, existe heterogeneidad en cuanto a la asignación del porcentaje de la evaluación sumativa que se destina a las actividades de aprendizaje que integran alguna acción investigativa. Por ende, se debe establecer una norma en cuanto a la asignación de porcentaje de la evaluación sumativa fijada a cada actividad de aprendizaje, en función de la carga académica que supone para el estudiante.

"La ECE debería contar con un equipo de gestión académica que coadyuve a articular los esfuerzos y que vele porque las asignaturas cumplan con los requerimientos para la consecución de los perfiles profesionales establecidos en cada carrera. Si bien los encargados de cátedra y encargados de programa tienen responsabilidades sobre la gestión de los cursos y del plan de estudios, actualmente, al menos en la $E C E$, no existe un mecanismo que verifique oportunamente la pertinencia de los modelos de evaluación y de los instrumentos de evaluación utilizados en cada asignatura, ni tampoco se 
coteja la pertinencia y suficiencia de las orientaciones de cursos brindadas a cada estudiante.

»De la idea anterior, se desprende la necesidad de establecer acuerdos mínimos en la carrera y de la ECE, en torno a los ejercicios académicos realizados por los estudiantes, como, por ejemplo, el estilo de citación y la elaboración de referencias, la divulgación de información respecto del plagio, la orientación de los estudiantes ( $y$, también, de los tutores) para que usen bien las bases de datos y las revistas electrónicas a las cuales tienen acceso en la universidad. En este sentido, hay que unificar alguna información en las orientaciones de los cursos, además de privilegiar actividades para que los estudiantes desarrollen, exitosamente, competencias básicas en sus labores académicas y sus TFG.

"Como parte del mejoramiento de la formación de la carrera, todos los estudiantes y tutores de la UNED deben recibir formación en estrategias de búsqueda y uso adecuado de recursos bibliográficos. Se garantizaría el acceso a investigaciones y artículos científicos que cumplan con mínimos de calidad para apoyar el desarrollo de las asignaciones y trabajos solicitados al estudiantado en las asignaturas; además, abordar con detalle el tema del plagio les permitiría a estudiantes y docentes establecer estrategias para prevenirlo y restringir el uso de recursos digitales de baja confiabilidad.

\section{BIBLIOGRAFÍA}

Alfaro, G. y Francis, S. (2009). Investigación y formación: Teoría y práctica de la investigación Educativa en la formación de Educadores: Manual de grado. San José: Universidad de Salamanca: AECI: Universidad de Costa Rica.
Acevedo, A. (2007). Sistema Educativo, educación preescolar. Recuperado el 01 de marzo del 2011, de <http://www.lajornadanet.com/diario/opinion_base/publicados/2007/enero/educaciónpre-escolar-9.html>

Asociación Mundial de Educadores Infantiles. (2006). La educación de la primera infancia: Reto del Siglo XXI. México: Trillas.

Campos, J. y Castro, D. (2010). Docente investigador de su quehacer en Costa Rica. Revista Centroamericana de Educación y Cultura Patria Grande, 1 (1). Recuperado el 03 de marzo del 2011, de http://www.ceducar.info/OJS/index. php/rpg/article/view/3/17

Campos, J. y Chinchilla, A. (2009). Reflexiones acerca de los desafíos en la formación de competencias para la investigación en educación superior. Revista Electrónica Actualidades Investigativas en Educación, 9 (2), pp. 1-20. Recuperado el 20 de agosto del 2010, de <http:// redalyc.vaemex.mx/src/inicio/ArtPdfRed. jsp?iCve $=44713058023>$

Cerda, H. (2007). La investigación formativa en el aula la pedagogía como investigación. Bogotá, Colombia: Magisterio.

Day, Ch. (2005). Formar docentes: cómo, cuándo y en qué condiciones aprende el profesorado. Madrid, España: Nárcea.

Egido, I. (2000). La Educación Inicial en el ámbito internacional: Situación y perspectivas en Iberoamérica y en Europa. Recuperado el 01 de marzo del 2011, de <http://www.rieoei.org/ riez2ao6.html>

Freire, P. (2004). Pedagogía de la autonomía. Madrid, España: Siglo XXI Editores.

Hidalgo, R., Solano, V., Valverde, C., Monge, I., Guerrero, M., Hernández, J., Rivera, G. y Valverde, H. (2010). Rediseño Plan de la Carrera: Diplomado, Bachillerato y Licenciatura en Educación Preescolar. San José, Costa Rica: Universidad Estatal a Distancia.

Mcmillan, J. H. y Schummacher, S. (2005). Investigación educativa. Madrid, España: Pearson.

Ministerio de Educación y Deportes. (2005a). Educación inicial. Bases curriculares. Recuperado el 
01 de marzo del 2011, de, de <http://www.oei. es/inicial/curriculum/bases_venezuela.pdf >

Ministerio de Educación y Deportes. (2005b). Educación inicial. Evaluación y planificación. Recuperado el 01 de marzo del 2011, de http://www. unicef.org/venezuela/spanish/educinic3.pdf

Morales, R. (2011). Aportes educativo, político y social en torno a la investigación con innovación en la UNED Costa Rica. Recuperado el o1 de marzo del 2011, de http://congreso.inie.ucr. ac.cr/memoria/documentos/1/Aportes\%20 educativo,\%2opolitico\%20y\%20social\%20 en\%2otorno\%20a\%2ola\%2oinvestigacionRoxana\%2oMorales.pdf

Restrepo, Bernardo. (2004). Formación investigativa e investigación formativa: acepciones y operacionalización de esta última. Recuperado el 25 de octubre del 2009, de http://hermesoft. esap.edu.co/esap/hermesoft/portal/home_1/ rec/arc_3529.pdf

Sevillano, M., Pascual, M., Bartolomé, D. (2007). Investigar para innovar en enseñanza. Madrid, España: Pearson Educación S. A.
Siverio, A. (2008). El Educador de la primera infancia: ¿formador, investigador, innovador? Revista Educación. Cuba, 124: pp. 23-26.

Stenhouse, L. (2003) La investigación y desarrollo del currículum. Madrid, España: Morata.

Universidad Estatal a Distancia (2004). Modelo Pedagógico. Recuperado el 03 de octubre del 2009, de <http://www.uned.ac.cr/paa/pdf/Materiales-autoev/24.pdf $>$

Universidad Estatal a Distancia (2008). Plan Académico 2008-2011. San José, Costa Rica: EUNED.

Venegas, M. E. (2009). ¿Cómo se forman los docentes para las escuelas en Centroamérica y República Dominicana. Consolidación de las acciones de mejoramiento de la formación inicial de docentes para la educación primaria en Centroamérica y República Dominicana. San José, Costa Rica: Coordinación Educativa y Cultural Centroamericana.

Venegas, M. E. (2008). Tendencias de la formación inicial de docentes. Revista Centroamericana de Educación. San José, Costa Rica: CECC, 3 (1), pp. 271-284. 